\title{
Saccadic eye movements and localization of visual stimuli
}

\author{
STEFAN MATEEFF \\ Institute of Physiology, Bulgarian Academy of Sciences, 1113 Sofia, Bulgaria
}

\begin{abstract}
Visual localization phenomena were studied before, during, and after a saccade. Light flashes of .5 and $9 \mathrm{msec}$ duration presented before and during the eye movement were mislocated in the saccade direction, the localization error being a time function. When the 9-msec duration stimulus and saccade did not overlap in time, a stripe was reported, when they did not, the stimulus was perceived as a point. If a long-duration stimulus moved perpendicularly to the saccade direction with the same "sigmoidal" velocity, a curvilinear trace was perceived, regardless of the linear trace of the image on the retina. A stimulus with stabilized retinal image was perceived as a stationary point during the saccade. A possible theory to deal with the data was suggested by modifying the algebra of outflow-inflow theories.
\end{abstract}

When a visual stimulus is presented, one of the tasks presented to our visual system is to answer the question, "Where is it?" Our everyday experience shows this task to be performed relatively well. What is more, usually we localize surrounding objects properly, no matter where we look. In spite of the fact that a shift of gaze results in changes of the loci of the retinal image, under normal conditions object localization remains constant. This property of the visual system is known as "position constancy."

Two of the explanations of position constancy are provided by the outflow and inflow theories, which are described briefly as follows. A center, or mechanism, which receives two "messages" is assumed to exist in the visual system. The first of these "messages" concerns the locus of the retinal image. The second provides information about the position of the eye. These two "messages" are somehow summated algebraically, and the resultant of this operation determines the perceived locus of the object. The difference between the two theories concerns the source of the information about eye position. According to the outflow theory (Holst, 1954; Holst \& Mittelstaedt, 1950), this "message" comes from a hypothetical neural center from which the command to turn the eye originates, and it is thought that the "message" is a copy of the command for the eye movement. According to the alternative inflow theory (Sherrington, 1918), eye muscle proprioception provides this information.

We will not deal with the outflow-inflow controversy in the present paper. The fact of importance

The author is greatly indebted to Professor L. Mitrani and to Dr. N. Yakimoff from the Institute of Physiology, Sofia, and to Dr. M. S. Smirnov from the Institute of Problems of Information Transmission, Moscow, for their fruitful participation in the discussion of the work and for their recommendations. for us is that both theories imply the same algebraic operations between information about the retinal locus of the object and about position of the eye. Thus, we shall consider them as one theory and call it "subtraction theory."

Several authors (Bischof \& Kramer, 1968; Matin, 1972; Matin \& Matin, 1972; Matin, Matin, \& Pearce, 1969; Matin, Matin, \& Pola, 1970; Matin \& Pearce, 1965; Monahan, 1972) have shown that a brief light stimulus presented during or just before voluntary saccadic eye movements is frequently mislocated by considerable amounts. The size of the error depends on the time at which the stimulus is presented relative to the saccade onset.

Matin, Matin, and Pearce (1969) and Matin, Matin, and Pola (1970) showed that if subtraction theory was to be viable enough to deal with their data it would have to be substantially modified. They assumed that the information about the eye movement did not parallel the actual course of the saccade. More than a simple mismatch of latencies between information regarding the retinal locus of the image and the position of the eye was required, however; they also found that it was necessary to assume that the information regarding the change in eye position extended over a much longer time than is taken by the saccade itself.

In the present paper, we develop a quantitative model involving a temporally extended signal regarding the eye movement and show that this predicts the data if we add several additional assumptions: (1) The information regarding events at a single retinal point is not processed separately at each moment; the localization center integrates information over a considerable length of time. (2) The localization center processes the information about the locus of each stimulated point on the retina separately from every other point. 


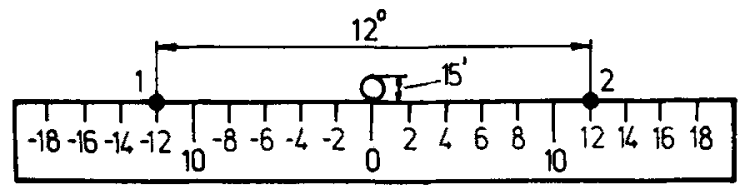

Figure 1. The scale with divisions; 1 angular degree $=2$ scale units. 1,2 = fixation points. The stimulus is labeled above the zero scale division.

\section{EXPERIMENT 1}

\section{Method}

The experimental arrangement (Figure 1) resembled that used by Bischof and Kramer (1968). The subject sat $57 \mathrm{~cm}$ in front of a screen of black paper. A horizontal scale with divisions was mounted on the screen at the subject's eye level. Two fixation points $12^{\circ}$ apart were placed on the scale. The experiment was carried out in darkness, with only the scale and the fixation points illuminated and clearly visible.

There was a circular hole, $15^{\prime}$ in diameter, in the black screen above the zero scale division. The cathode-ray tube of a monitor was placed on the rear of the screen. The electron beam was focused to produce a bright flash on the hole. The parameters of the flash were determined by settings of a square-wave generator which had provisions for varying the delay and duration of single rectangular pulses. The output of this generator was fed to the intensity-modulating input of the monitor. Thus, the stimulus consisted of a circle of light of about $.5 \mathrm{msec}$ duration' and was always presented above the zero scale division, straight in front of the subject (Figure 1).

The screen and the scale were viewed binocularly. The subject's eye movements were registered photoelectrically (Mitrani, Mateeff, \& Yakimoff, 1970). The light from an incandescent bulb passed through an infrared filter and illuminated the right eye. After reflection from the eye, the light fell on two appropriately placed phototransistors connected in a bridge circuit. The output was thus related to the rotation of the eye in the horizontal plane. This was fed to an oscilloscope, and the experimenter was able to observe the movement visually. The smallest eye movement that could be registered by this method was about $10^{\prime}-15^{\prime}$.

The subject was instructed to perform a saccade from the left fixation point to the right one immediately after an auditory signal. The square-wave generator was started simultaneously with the signal. When the delay of the generator output was longer than the subject's reaction time for saccade performance, the stimulus was presented after the saccade onset, and vice versa. The experimenter varied the stimulus delay arbitrarily during the experiment. Thus, due to the natural variations of the reaction time, the stimulus was presented practically randomly in time.

The signal from the eye-movement registering system triggered a discriminator at the very beginning of the saccade. The time interval between the stimulus and the pulse from the discriminator was measured by two electronic chronometers (made in Bulgaria). The first of these measured the time interval when the stimulus appeared before the saccade onset, the second, when the saccade onset came before the stimulus. Thus, the moment of stimulus presentation before as well as after the saccade onset was determined with a precision of about 1-2 msec.

After the saccade performance, the subject had to report verbally the scale division above which he had seen the stimulus. Reports of "I did not see" or "I cannot say" were allowed. The experimenter monitored the correct performance of the eye movement on the oscilloscope. Trials were rejected when, instead of a single $12^{\circ}$ saccade, a smaller one or other saccades occurred before or after the main eye movement. After each performance, the experimenter registered the subject's report and the moment of stimulus presentation, determined as described above.

Three subjects with normal vision, aged 20-25 years, participated in the experiment. About 600 successful trials were carried out with each of them. The subjects were told that the stimulus would be presented at various scale divisions, and until the end of the experiment they did not guess that its place of presentation was, in fact, always the same.

\section{Results}

The time interval of about $150 \mathrm{msec}$ before to $60 \mathrm{msec}$ after the saccade was investigated. It was divided into subintervals of $10 \mathrm{msec}$, and the subject's reports to stimuli presented in each subinterval were averaged and the $95 \%$ confidence limits were determined.

The results for all subjects were shown in Figures $2 a, 2 b$, and $2 c$. The moment of stimulus presentation, $t$, is plotted in milliseconds on the abscissa, the zero point being the saccade onset. The subinterval averages of the subject's reports, $y^{*}$, measured in angular degrees from the zero scale division are plotted on the ordinate.

The data show that a stimulus presented in the time interval $[-120 \mathrm{msec}, 60 \mathrm{msec}]$ is mislocated mainly in the direction of the saccade. The maximum mislocation error of $3^{\circ}-5^{\circ}$ occurs at the saccade onset, such that such stimuli are localized near the right fixation point. Long before and after the saccade, the subjects reported the stimulus always to be above the zero division.

\section{EXPERIMENT 2}

\section{Method}

The arrangement of the second experiment was the same as that in Experiment 1, but the stimulus duration was $9 \mathrm{msec}$. The subjects were told they would be presented with a horizontal light stripe above the scale during each saccade performance, and the length and locus of the stripe would vary randomly from trial to trial. Their task was to localize the stripe by reporting verbally the numeral of the scale division that coincided with each of the two ends of the stripe. If the subjects perceived the stripe as being reduced to a point, they were to report only one scale division.

Three subjects with normal vision, aged $20-25$, participated in the experiment; about $\mathbf{4 0 0}$ successful trials were carried out with each of them.

\section{Results}

The results from Experiment 2 for all subjects are shown in Figures $3 a, 3 b$, and $3 c$. They were averaged in subintervals, in a fashion similar to that of the previous experiment. The moment of stimulus onset, $t$, is plotted in milliseconds on the abscissa, the subject's reports, $y_{1}^{*}$ and $y_{2}^{*}$, for the location of the left and the right end of the stripe, respectively, are plotted on the ordinate.

The data show that the subjects perceive the stimulus correctly only when it is presented beyond the time interval $[-100 \mathrm{msec}, 60 \mathrm{msec}]$. If a stimulus 

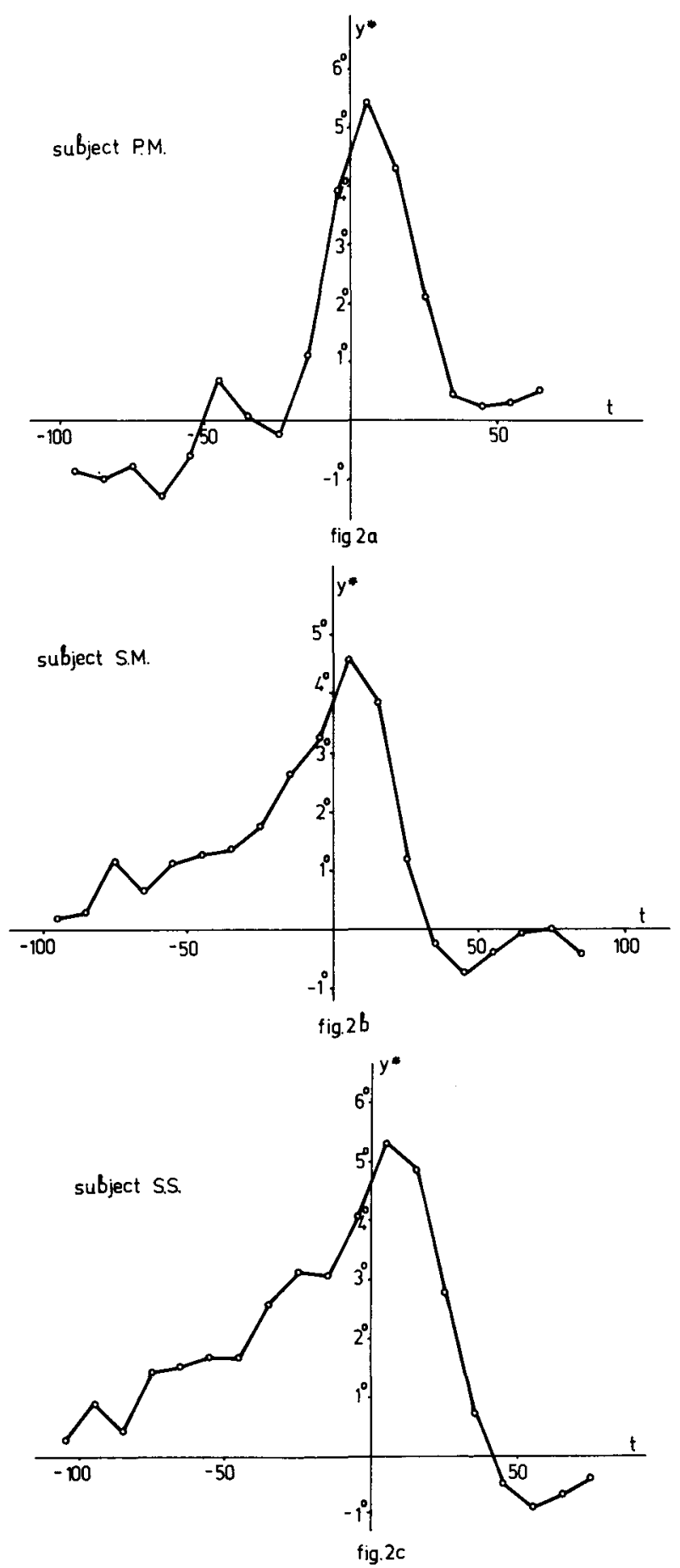

Figure 2. (a, b, and c) Data obtained from three subjects in Experiment 1. The dependence of the perceived stimulus position, $y^{*}$, on the moment of presentation, $t$, is shown. The $95 \%$ confidence limits are $\pm .5^{\circ}-1^{\circ}$.

presented in this interval does not overlap the eyemovement duration, it is perceived as a point and is mislocated in the saccade direction. Thus, the subjects report only one numeral and the values of $y_{1}^{*}$ and $y_{2}^{*}$ coincide. If the stimulus duration does overlap the eye-movement duration, it is perceived as a stripe mislocated in the direction of the saccade. The time course of the curves $y_{1}^{*}(t)$ and $y_{2}^{*}(t)$ resembles the time course of $y^{*}(t)$ obtained in Experiment 1 (Figures 2a, 2b, and 2c).
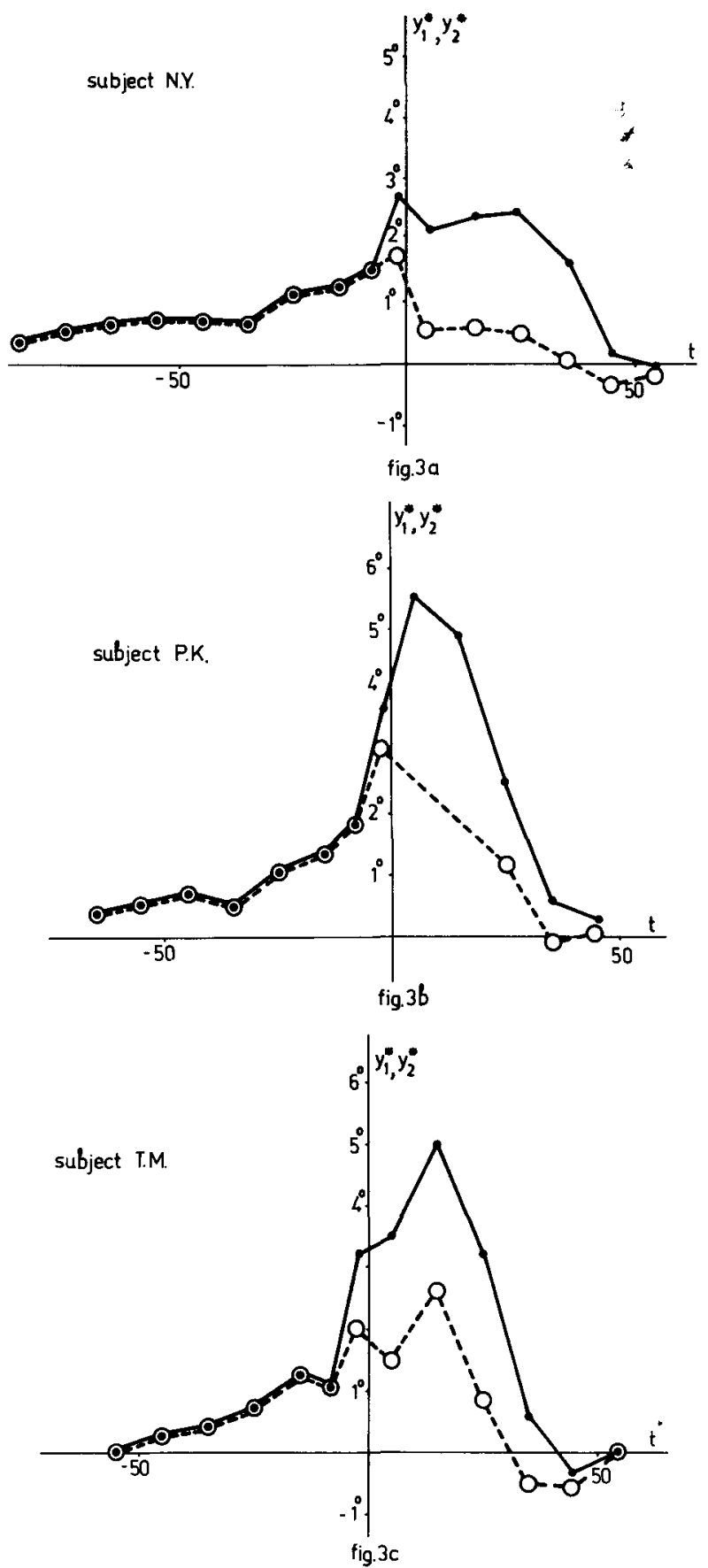

Figure 3. (a, b, and c) Data obtained from three subjects in Experiment 2. The perceived position, $y_{1}^{*}$, of the left end of the stripe is drawn with dashed lines, and that of the right end, $y_{2}^{*}$, with solid lines. When the stimulus duration does not overlap the saccade duration, $y_{2}^{*}$ and $y_{1}^{*}$ coincide. The $95 \%$ confidence limits are $\pm .5^{\circ}-1^{\circ}$. 


\section{DISCUSSION OF EXPERIMENTS 1 AND 2}

The results from Experiment 1 are in keeping with the findings of Bischof and Kramer (1968), Matin (1972), and Monahan (1972), who also established mislocation of brief light stimuli presented both before and during the saccade.

As mentioned above, the phenomenon of mislocation can be explained from the point of view of subtraction theory by assuming that the information about eye position the visual system receives is incorrect. The data from Experiment 1 permit us to try to infer the quantitative characteristics of this information. For this purpose, we shall introduce several terms.

Let us consider the cyclopean eye $e^{2}$ of a subject with fixed head. In Figure 4, the center of the eyeball is labeled $\mathrm{C}$, the direction straight ahead of the subject is labeled $\mathrm{Y}$, and the axis of the eye is $\mathrm{R}$. The locus of any stimulus on the scale can be determined by: (1) an angle, $y$, measured from the $\mathrm{Y}$-axis, and (2) an angle, $r$, measured from the R-axis. The letters $y$ and $r$ can be considered angular coordinates of the stimulus in two coordinate systems connected with the subject's head and eye, respectively. Thus, $r$ represents the position of the stimulus image on the retina measured in angular degrees from the fovea. The relation between $y$ and $r$ is

$$
\mathrm{y}=\mathrm{r}+\alpha,
$$

where $\alpha$ is the angle of rotation between $\mathrm{R}$ and $\mathrm{Y}$, i.e., the angle of eye rotation. In Figure 4, the positive direction is taken to be clockwise, i.e., the direction of the saccade from left to right.

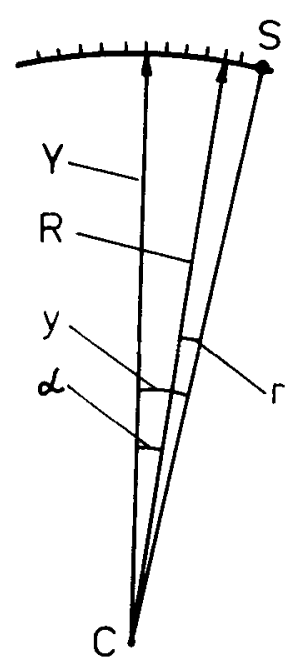

Figure 4. $S=a$ stimulus above the scale, $Y=$ direction straight ahead of the subject, $R=$ the eye axis, $C=$ the cyclopean eyeball center, $y=$ angular coordinate of the stimulus towards the $Y$-axis, $r=$ angular coordinate of the stimulus towards the $\mathbf{R}$-axis, and $\alpha=$ angle of eye rotation.
We have already designated the subject's report $y^{*}$. When stimulus localization is correct, $y=y^{*}$.

We now describe the subtraction theory in the terms we have introduced above: The mechanism of localization receives information about the stimulus image locus on the retina, $r$, and the angle of eye position, $\alpha$. Using the terminology of Matin (1972), we shall call the first "message" retinal signal (RS) and designate it $\mathrm{r}$ ". We shall call the second "message" extraretinal signal (ERS) and designate it $\alpha^{*}$. Then, according to the suggestions of the subtraction theory, the subjectively determined location of the stimulus $\mathrm{y}^{*}$ will be

$$
y^{*}=r^{*}+\alpha^{*}
$$

Equation 2 has the same form as Equation 1, but it has other components. It refers to the mode of information processing in the localization center.

Let us consider the term "signal" in more detail. Here this term means neither nerve cell discharge nor number of nerve impulses per second, but information about angular distance on the retina, or angle of eye rotation. When we speak about the "value" of the signal, we refer to the actual value of the distance on the retina, or angular extent of eye rotation.

The time course of $y^{*}$ was obtained in Experiment 1 . Keeping in mind that in our experiments the stimulus image was $\pm 6^{\circ}$ away from the fovea at most, we assume that the RS represents its retinal locus correctly, i.e., we always have $\mathrm{r}^{*}=\mathrm{r}$. Hence,

$$
a^{*}=\mathrm{y}^{*}-\mathrm{r}^{*}=\mathrm{y}^{*}-\mathrm{r} .
$$

The time course of $\alpha^{*}$ can be determined most easily in a graphical way. The time is plotted on the abscissa in Figures 5a, 5b, 5c, and 5d, the zero point being the saccade onset. Figure 5 a represents the idealized time course of $y^{*}$ obtained in Experiment 1 (Figures $2 a, 2 b$, and $2 c$ ). In Figure $5 b$, the time course of $\mathrm{r}^{*}$ is plotted on the ordinate. This curve represents approximately the position, $r$, of a brief stimulus relative to the visual axis, $R$ (and thus also relative to the fovea), the stimulus being presented at the moment, $t$, before or after the onset of the $12^{\circ}$ saccade. The duration of a $12^{\circ}$ saccade is taken to be $50 \mathrm{msec}$, but if it is taken to be 40 or $60 \mathrm{msec}$, the results would not change essentially. The result of the point-to-point subtraction of the curves $y *(t)$ and $r^{*}(t)$ (according to Equation 3 ) is given in Figure 5c. If a subtraction theory is accepted, an ERS shaped as shown in Figure 5c is necessary to explain the mislocation obtained in Experiment 1. For comparison, a typical S-shaped time course, $\alpha(t)$, of a $12^{\circ}$ saccade is shown in Figure $5 \mathrm{~d}$. It can be seen that the ERS determined above is monotonically related to the saccade, but does not coincide with it. For example, the mechanism of localization might 

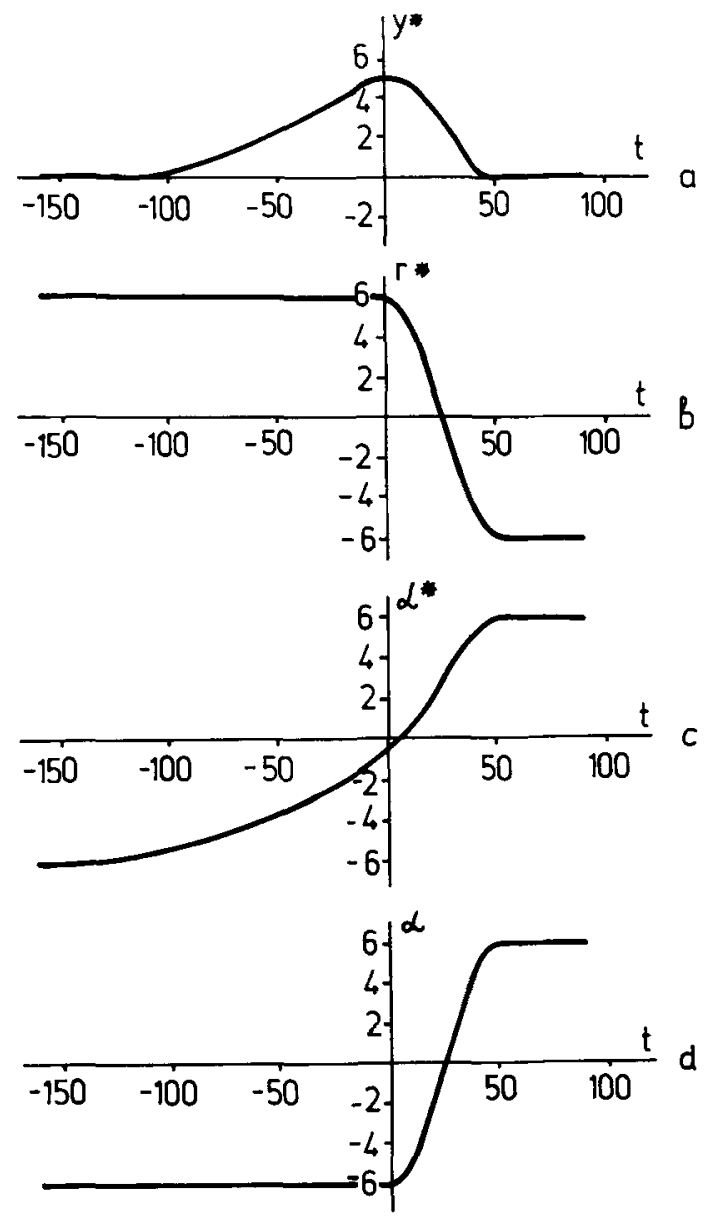

Figure 5. (a) An idealized time course of the perceived stimulus position, $y^{\star}$, according to the data from Experiment 1. (b) An idealized time course of $r^{*}$ according to the typical saccade shape. (c) The time course of the ERS $\alpha^{*}$ obtained by point-to-point subtraction between $y^{\star}$ and $r^{*}$. (d) A typical time course of $a 12^{\circ}$ saccade.

receive information that the eye has already been turned before the actual saccade. The difference between $\alpha^{*}(\mathrm{t})$ and $\alpha(\mathrm{t})$ is equal to the localization error.

In Experiment 1, we found a mislocation in the direction of the saccade. Bischof and Kramer (1968), however, reported that it was possible for the stimulus to be located in the direction opposite to the saccade. These data could be accounted for by a simple assumption. Let us imagine an ERS of the same shape as shown in Figure 5c. Let us assume that this signal arrives in the localization center following some delay, as expressed in Figures $6 a$ and $6 b$, where the time courses of $\mathrm{n}^{*}$ and $a^{*}$ are drawn, $\alpha^{*}$ being delayed relative to $r^{*}$. The point-to-point addition of the two curves according to Equation 2 results in a curve for $\mathrm{y}^{*}$ (Figure $6 \mathrm{c}$ ) with negative values, i.e., in mislocation in the direction opposite to the saccade. The curve in Figure $6 \mathrm{c}$ resembles exactly the data obtained by Bischof and Kramer (1968, Figures 5 and 6 on page 195).
Of course, this is only a hypothesis to explain why the mislocation can be either in the saccade direction or in the opposite one. We are not in a position to suggest a method for control of the ERS delay. The time mismatch between RS and ERS might be a characteristic, specific for each subject. In any case, we failed to observe mislocation opposite to the saccade in our experiments.

The data from Experiment 2, where the stimulus duration was relatively longer, are in general accordance with the findings of Kennard, Hartmann, Kraft, and Glaser (1971). Keeping in mind the obtained course of the ERS, we can try to apply the ideas of the subtraction theory to explain these data.

Let us designate the onset and the end of the stimulus $t_{1}$ and $t_{2}$, respectively. Let us consider this stimulus as an infinite set of instantaneous stimuli presented successively in the time interval $\left[t_{1}, t_{2}\right]$. We can apply Equation 2 for each of them, taking into account the time courses of $r^{*}$ and $\alpha^{*}$ in Figure 5. Thus, we have, for $\mathrm{y}^{*}\left(\mathrm{t}_{1}\right)$ and $\mathrm{y}^{*}\left(\mathrm{t}_{2}\right)$,

$$
\begin{aligned}
& y^{*}\left(t_{1}\right)=r^{*}\left(t_{1}\right)+\alpha^{*}\left(t_{1}\right), \\
& y^{*}\left(t_{2}\right)=r^{*}\left(t_{2}\right)+\alpha^{*}\left(t_{2}\right) .
\end{aligned}
$$

When the stimulus is presented just before the sac-
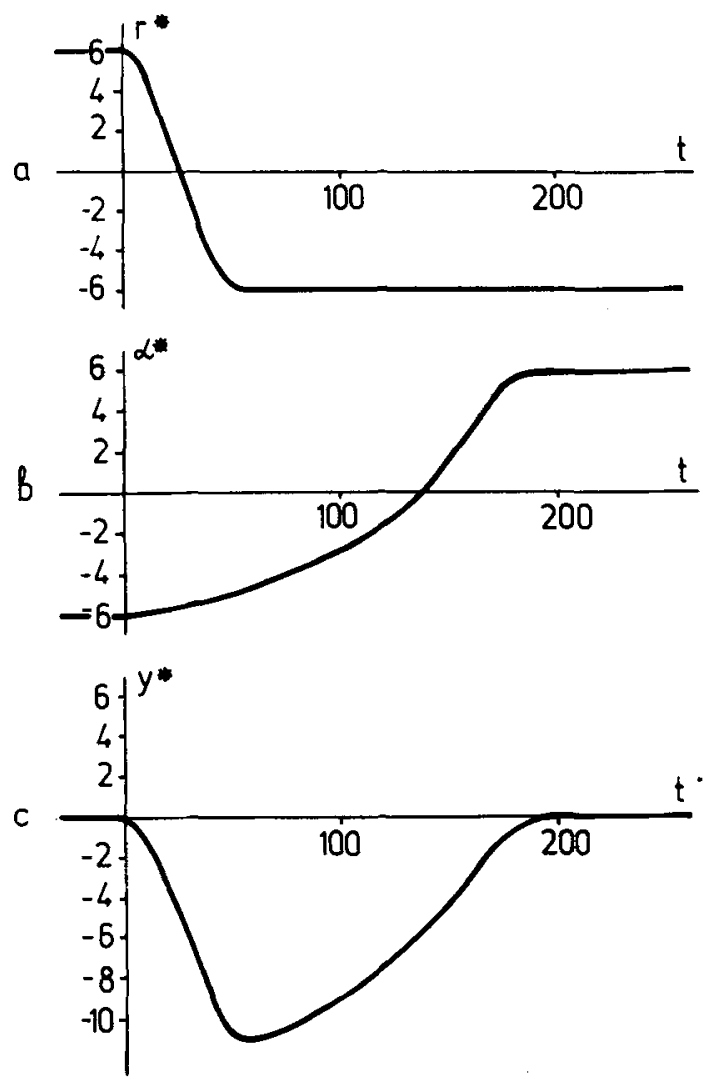

Figure 6. (a and b) Time course of $r^{*}$ and $\alpha^{*}$ shaped as in Figure $5, \alpha^{*}$ being delayed in respect to $r^{*}$. (c) The result of the point-to-point summation of $r^{\star}$ and $\alpha^{\star}$. 
cade, its locus on the retina remains constant, thus $\mathrm{r}^{*}\left(\mathrm{t}_{1}\right)=\mathrm{r}^{*}\left(\mathrm{t}_{2}\right)$. The ERS, however, is a time function, thus $a^{*}\left(\mathrm{t}_{1}\right) \neq \alpha^{*}\left(\mathrm{t}_{2}\right)$. Hence, we have $\mathrm{y}^{*}\left(\mathrm{t}_{1}\right) \neq \mathrm{y}^{*}\left(\mathrm{t}_{2}\right)$, or, more exactly, each stimulus of the imaginary set of stimuli with equal retinal locus should have been localized at various places relative to the observer, in accordance with the value of the ERS at the moment $t$, where $t_{1} \leqslant t \leqslant t_{2}$. That is why the subject might always be expected to report a stripe with ends $y^{*}\left(t_{1}\right)$ and $y^{*}\left(t_{2}\right)$, respectively.

It is important that no such reports were obtained in Experiment 2 when the stimulus and saccade did not overlap in time. In order for a stripe to be reported, it was necessary that the stimulus be present during at least some portion of the saccade (i.e., that there be a blur on the retina). Thus, the interpretation given above is not adequate to deal with this result. Apparently, we cannot treat a long-duration stimulus presented to a fixed retinal locus as a set of brief independent flashes presented sequentially.

It is possible to modify the subtraction theory, however, to deal with the above results. Let us consider the following equation:

$$
t^{*}=t_{1}+k\left(t_{2}-t_{1}\right),
$$

where $t_{1}$ and $t_{2}$ are the moments of the onset and the end of the stimulus, $\mathrm{k}=$ constant, $0<\mathrm{k}<1$. We shall call $\mathrm{t}^{*}$ generalized moment of stimulus presentation (GMSP). Let us assume that a stimulus of any given duration and constant retinal position is localized according to the equation:

$$
\mathrm{y}^{*}=\mathrm{r}^{*}+\alpha^{*}\left(\mathrm{t}^{*}\right)
$$

where $\mathrm{y}^{*}$ is the reported location, $\mathrm{r}^{*}$ is the RS value, and $\alpha^{*}\left(t^{*}\right)$ is the ERS value at the moment $t^{*}$.

The introduction of the GMSP is a means of reconciling the discrepancy between the experimental data and the assumption that the stimulus of long duration is processed as a set of successive instantaneous stimuli. The application of Equation 5 to the above situation-a stimulus of long duration at a constant retinal locus-does not result in a perception of a stripe, but of a single point-like stimulus mislocated according to the ERS value at the moment $t^{*}$. On the other hand, when the stimulus is actually instantaneous with a moment of presentation, $t$, we have $\mathrm{t}_{1}=\mathrm{t}_{2}=\mathrm{t}$ and Equation 5 becomes Equation 2.

When the long-duration stimulus does overlap the saccade duration, the image of the stimulus is a stripe on the retina. We now assume this stripe to be a spatial set of point-like stimulus images, each of which is instantaneous and characterized by a retinal locus, $r$, as well as a moment, $t$, at which the image passes over this locus. For each image of this set, we can apply Equation 2, and we can predict that the subject perceives a stripe with ends $y_{1}^{*}$ and $y_{2}^{*}$ as follows:

$$
\begin{aligned}
& y_{1}^{*}=r^{*}\left(t_{1}\right)+\alpha^{*}\left(t_{1}\right), \\
& y_{2}^{*}=r^{*}\left(t_{2}\right)+\alpha^{*}\left(t_{2}\right) .
\end{aligned}
$$

Here $\mathrm{r}^{*}\left(\mathrm{t}_{1}\right) \neq \mathrm{r}^{*}\left(\mathrm{t}_{2}\right), \alpha^{*}\left(\mathrm{t}_{1}\right) \neq \alpha^{*}\left(\mathrm{t}_{2}\right)$, and hence $\mathrm{y}_{1}^{*} \neq \mathrm{y}_{2}^{*}$. This is essentially what was obtained in Experiment 2 .

Thus, in order to account fully for the results from Experiment 2 in terms of a subtractive theory, we introduced the GMSP $t^{*}$ and applied Equation 5 for each stimulated point on the retina. These are assumptions about the way the localization mechanism might process the information, and they should be added to the subtraction theory, whether it be inflow or outflow.

In order to test the plausibility of our assumptions, we used them for making some further experimental predictions.

\section{LOCALIZATION OF STIMULI OF INFINITELY LONG DURATION}

Let a stimulus be presented straight in front of the subject $(y=0)$ and let the saccade be performed between two fixation points $6^{\circ}$ to the left and $6^{\circ}$ to the right of the stimulus, respectively, i.e., with presentation conditions the same as in the previously described experiments. Let us analyze the stimulus image on the retina. Before the saccade, the gaze is directed to the left fixation point $\left(\alpha=-6^{\circ}\right)$, the stimulus image locus on the retina is kept fixed, and $r=6^{\circ}$ (see Figures $5 \mathrm{~b}$ and $5 \mathrm{~d}$ ). Let us calculate $\mathrm{t}^{*}$. Substituting $t_{1}=-\infty$ and $t_{2}=0$ (the zero point is the saccade onset) in Equation 4, we obtain $t^{*} \rightarrow-\infty$. As seen in Figure $5 \mathrm{c}$, long before the eye movement, the information about eye position is correct, i.e., $\alpha^{*}(-\infty)=\alpha(-\infty)=-6^{\circ}$. Applying Equation 5, we have $\mathrm{y}^{*}=\mathrm{r}^{*}+\alpha^{*}\left(\mathrm{t}^{*}\right)=6^{\circ}-6^{\circ}=0$. Hence, this part of the retinal picture is expected to lead to a perception of a point-like stimulus placed straight in front of the subject.

During the eye movement, the stimulus image "draws" a $12^{\circ}$ stripe on the retina, from $r\left(t_{a}\right)=6^{\circ}$ to $r\left(t_{b}\right)=-6^{\circ}$. Here the very beginning of the saccade is designated $t_{a}\left(t_{a} \approx 0\right)$ and its end, $t_{b}$. According to one of our assumptions, this stripe is to be considered as a set of contiguous images of instantaneous point-like stimuli on the retina, and Equation 2 is to be applied for each of them. Thus, a stripe should be perceived with ends located, respectively, at $\mathrm{y}^{*}\left(\mathrm{t}_{\mathrm{a}}\right)$ and $\mathrm{y}^{*}\left(\mathrm{t}_{\mathrm{b}}\right)$. From Figure 5 , we have $\mathrm{r}^{*}\left(\mathrm{t}_{\mathrm{a}}\right)=6^{\circ}$ and $\alpha^{*}\left(\mathrm{t}_{\mathrm{a}}\right) \approx-1^{\circ}$. Substituting in Equation 2 , we receive $y^{*}\left(t_{a}\right) \approx 5^{\circ}$. By similar calculations, 
we obtain $y^{*}\left(t_{b}\right)=0$, because $r^{*}\left(t_{b}\right)=-6^{\circ}$ and $\alpha^{*}\left(t_{b}\right)=6^{\circ}$.

After the termination of the eye movement, the stimulus image is point-like and stationary again. In this case, $t_{1}=t_{b}$ and $t_{2}=\infty$. Substituting in Equation 4 , we have $t^{*}=\infty$. It is clear from Figure $5 c$ that when $\mathrm{t} \rightarrow \infty$, then $\alpha^{*}=6^{\circ}$ and $\mathrm{r}^{*}=-6^{\circ}$. Substituting these values in Equation 5, we obtain $y^{*}=0$. Therefore, the last part of the retinal picture again leads to the perception of a point-like stimulus presented straight ahead to the subject.

The localization of the stimulus both before and after the saccade proves to be the same, $y^{*}=0^{\circ}$. Moreover, a perception of a horizontal stripe is expected. Its left end is to be located straight ahead, and the right one about $5^{\circ}$ in the direction of the saccade goal.

For the above calculations, we used the data from Experiment 1, shown in Figure 5. As we have pointed out, other results are also possible (Bischof \& Kramer, 1968). If this were the case, we should have used the time course of $\mathrm{r}^{*}$ and $\alpha^{*}$ shown in Figure 6, and a perception of a stripe from the center to the left would have been predicted.

One can easily observe the predicted stripe by performing saccades in front of a point-like light source against a dark background. The first to report this stripe was Mach (1885). When the source is modulated with a frequency of $100-300 \mathrm{~Hz}$ instead of a stripe, a string of light flashes is perceived. The latter phenomenon is known as the "intermittent light illusion" (Matin, Matin, Pola, \& Kowal, Note 1). It is clearly visible that the stripe or string length is much shorter than the saccade size, regardless of the fact that the stimulus trace length on the retina is exactly equal to the saccade angular size. We suggest that perceptual shortening of the stripe results from the characteristics of the ERS and the mode of information processing in the localization center. ${ }^{3}$

Obviously, if the information about eye position was precisely correct, i.e., $\alpha^{*}=\alpha$ for each $\mathrm{t}$, no stripe would have been perceived. Our theory thus suggests that this phenomenon is a manifestation of incomplete position constancy during eye movements: the perception is a compromise between the actual stimulus configuration and the retinal picture.

An interesting fact emerges from the above calculations. The part of the retinal image corresponding to the time before the saccade leads to a veridical perception $-y^{*}=0$. On the other hand, $y^{*}\left(t_{a}\right)$ was found to be about $5^{\circ}$ to the right. Hence, a "point of disruption" of perception exists at the very beginning of the saccade. To demonstrate this "disruption of perception," we carried out the following experiment.

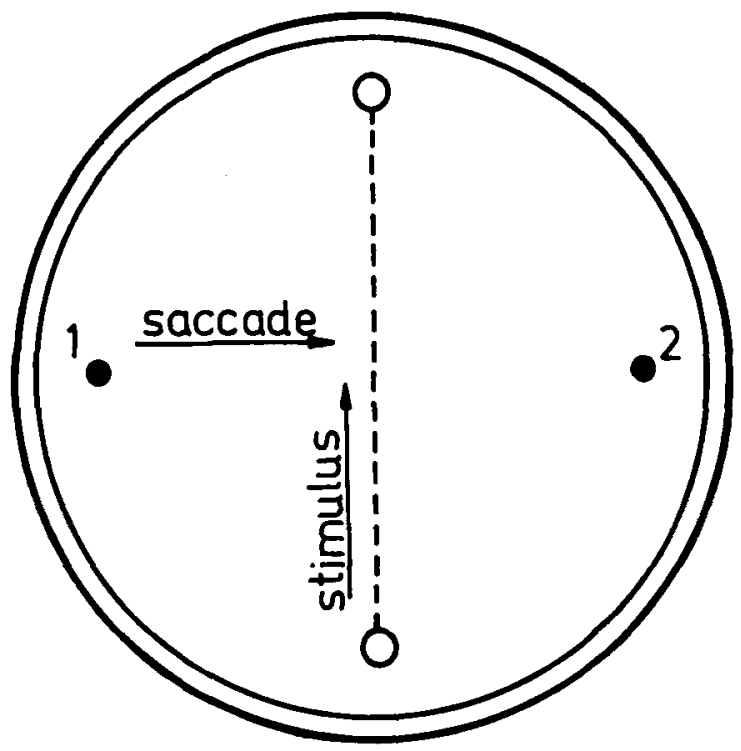

Figure 7. The oscilloscope screen with the fixation points 1 and 2 mounted on it. The electron beam motion in synchrony (i.e., with the same "sigmoidal" velocity) with the saccade is drawn with the dashed line.

\section{EXPERIMENT 3}

\section{Method}

The subject sat in a dark room in front of an oscilloscope screen. Two light fixation points, $12^{\circ}$ apart, were mounted on it. The electrical signal from the eye-movement-registering system was fed to the Y-input of the oscilloscope. When the subject looked at the left fixation point, the electron beam was $6^{\circ}$ below the point level. When an eye movement from the left to the right fixation point was executed, the beam moved synchronously (i.e., with the same "sigmoidal" velocity) with the eyes to a new position $6^{\circ}$ above the point level. Figure 7 shows the oscilloscope screen, the fixation points, and the trace of the electron beam. The subject performed several saccades from the left point to the right point and then was required to describe the picture he had seen. He either pointed with the finger on the screen or drew the trace on paper.

\section{Results}

The 10 subjects who participated in the experiment all described approximately the trace drawn in Figure 8. Before the saccade, the electron beam was localized at point $A$, which was its actual position. During the saccade, a light curve was perceived. Its lower end was point B-"somewhere on the right"and its higher end was point $C$, i.e., the actual position of the beam after the saccade. The subjects localized point $B$ to be $4^{\circ}-5^{\circ}$ to the right of point $A$.

Under the conditions of Experiment 3, the stimulus trace on the retina is a straight diagonal line connecting the initial and the final image loci. Due to the inherent properties of the localization mechanism, a distorted stimulus trace is perceived and the predicted "disruption of perception" between point $A$ and point $B$ occurs. Here point $A$ corre- 


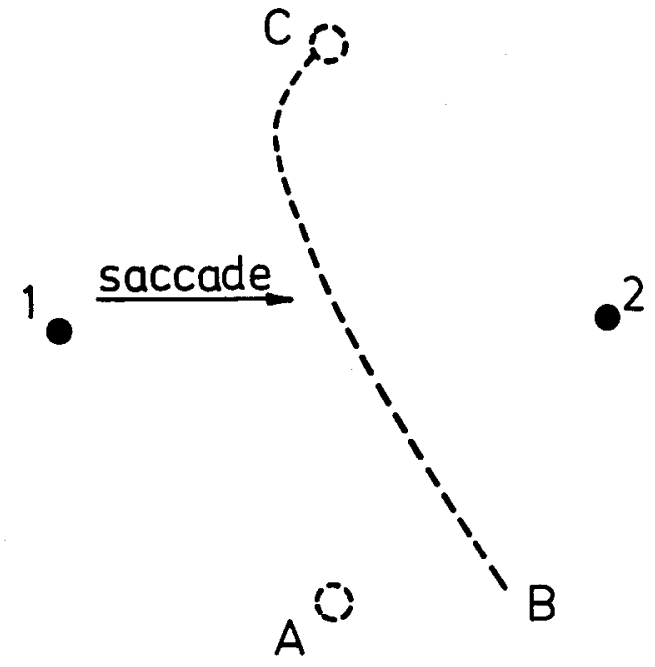

Figure 8. An example of a subject's perception in Experiment 3. $1,2=$ fixation points, $A$ and $C=$ perceived position of the beam before and after the saccade, and $\mathrm{BC}=$ the trace perceived during the saccade.

sponds to the stimulus image on the retina before the saccade and point $B$ corresponds to the image at the very beginning of the saccade, at the moment $t_{a} \sim 0$.

Kennard, Hartmann, Kraft, and Glaser (1971) reported similar observations. In their experiments, the stimulus moved with constant velocity perpendicularly to the saccade. The stimulus image was not immobile on the retina before the saccade, and hence Equation 4 was not to be applied. This fact could explain why the authors did not observe the above described "disruption of perception."

The perceived trace of stimulus motion under the conditions of Experiment 3 and of the experiments of Kennard et al. (1971) coincides neither with the retinal nor with the actual trace. It is perceived to be somewhere between them. The phenomenon is again a manifestation of incomplete constancy.

\section{LOCALIZATION UNDER CONDITIONS OF STABILIZED RETINAL IMAGE}

Let us imagine the following situation. The stimulus is visible only during the saccade and its retinal image is always stabilized in the fovea. Let us designate the saccade duration $T$. The stimulus is presented from the moment $t_{1}=t_{a} \approx 0$ to $t_{2}=t_{b} \approx T$. As the stimulation is always foveal, the RS value is $\mathrm{r}^{*}=0^{\circ}$. Let us calculate the localization in this case. For $t^{*}$, we have

$$
t^{*}=t_{1}+k\left(t_{2}-t_{1}\right)=0+k(T-0)=k T,
$$

and applying Equation 5

$$
\mathrm{y}^{*}=\mathrm{r}^{*}+\alpha^{*}\left(\mathrm{t}^{*}\right)=\alpha^{*}(\mathrm{kT})
$$

Hence, a point-like stimulus should be localized somewhere between the fixation points, because $\mathrm{y}^{*}=\alpha^{*}(\mathrm{kT})=$ constant and $-6^{\circ} \leqslant \alpha \leqslant 6^{\circ}$. In order to test this prediction, we carried out the following experiment

\section{EXPERIMENT 4}

\section{Method}

The subject sat in front of a dark translucent screen on which two light fixation points, $12^{\circ}$ apart, were mounted horizontally. The stimulus consisted of a circle of light, $.5^{\circ}$ in diameter, and was projected by an optical train from the rear of the screen. The light beam of the optical train was reflected onto a mirror mounted on a pen motor. The signal from the eye-movementregistering system was amplified and fed to the pen motor, causing rotation of the mirror and displacement of the stimulus between the fixation points. By appropriate adjustment of the experimental arrangement, a satisfactory synchronization of the stimulus and eye movement was provided. Thus, the retinal image of the stimulus was stabilized in the fovea during a $12^{\circ}$ saccade. Two sheets of black paper were placed behind the screen in such a way that the stimulus was visible only during the saccade (Figure 9). After performing several saccades from left to right, the subject was required to describe how he perceived the stimulus and to localize it on the screen by pointing with the finger.

\section{Results}

The three subjects who participated in the experiment reported perceiving the stimulus as an immobile spot placed about $8^{\circ}-9^{\circ}$ to the right of the left fixation point. In Figure 9, the approximate perceived stimulus locus is labeled $\mathrm{S}$.

This result is in keeping with the findings of Holly (1975) that a stimulus appears as a stationary dot when it moves at the same average velocity as the eyes.

The experiment is not only a verification of our prediction and confirmation of the validity of Equation 5 , but also shows that the coefficient, $k$, in Equation 4 is actually constant in respect to time. Apparently, if $k=k(t)$, then $y^{*}=\alpha^{*}(k T) \neq$ constant and the observer might be expected to perceive a stripe.

If the exact time course of $\alpha^{*}$ was known in Experiment 4, we could have determined the value of $k$ from the equation $y^{*}=\alpha^{*}(k T)$. Determining $\alpha^{*}(t)$ is impossible in this short experiment, but if we assume that the course of $\alpha^{*}(t)$ is the same as that shown in Figure $5 \mathrm{c}$, the coefficient $\mathrm{k}$ becomes about .8.

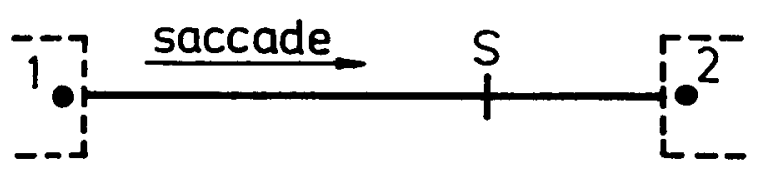

Figure 9. Elements of the arrangement of Experiment 4. 1,2 = fixation points. The stimulus motion in synchrony with the saccade is drawn with the solid line, the sheets of black paper behind the screen are drawn with dashed lines. The perceived stimulus position is labeled $S$. 


\section{THE SCALE AS A VISUAL STANDARD}

An important problem in the analysis of the localization phenomena is whether the scale could be considered a "stable"' standard, i.e., a standard stimulus which is not influenced by the operation of ERS.

To point to a stable standard would mean that there exists a world of "true" locations, and in spite of eye movements this standard always represents one (or more) of these locations. As suggested by Matin, Matin, and Pearce (1969), it is very difficult to point to a visual standard which would not be influenced by the ERS. In their experiments, the subjects reported on the visual direction of a test flash relative to a fixation target viewed and extinguished before the saccade. The authors consider the fixation target as a standard, but they assume the possibility "that the ERS operates on the memory of the visual direction of the fixation target instead of the retinal signal arising from the flash.... The test flash then provides the standard direction against which this memory is compared" (Matin, Matin, \& Pearce, 1969, page 78).

In the present experiments, the subject locates the test stimulus relative to a continuously illuminated scale (or to two steady light fixation points in Experiments 3 and 4). During the saccade, the scale divisions are blurred, and most probably the subject compares the perceived test stimulus locus only with the perceived scale position before and/or after the eye movement. On the one hand, the very essence of position constancy is that localization of normally illuminated objects remains constant when voluntary saccades occur. On the other hand, we found theoretically that a stimulus of infinitely long duration (such as the continuously illuminated scale) was mislocated only during the saccade and not before and/or after it. Thus, scale localization before and/ or after the saccade is the same, it corresponds to reality, and it does not depend on the moment of stimulus presentation. That is why we consider the scale to be an adequate visual standard.

The use of different standards results in quantitative differences between the data of Matin \& Matin (1972) and our data. Nevertheless, our findings (Figure 5) are in qualitative agreement with Matin's conclusions about the nature of ERS, namely: the ERS does not parallel the saccade course, but its asymptotic value is approximately equal to the saccade length.

\section{CONCLUSIONS}

The present work shows that the classical outflow theory is not sufficient to account for several phenomena connected with localization of stimuli during sac- cadic eye movements. In order to explain all the data obtained, three additional assumptions are necessary.

The first assumption is that the ERS does not parallel the actual saccade course. In Experiment 1, an example of the ERS time course was obtained.

The second assumption is that the RS related to the locus of a single point on the retina is not processed separately at each moment of time. To localize a long-duration stimulus with point-like retinal image, the localization center "takes into account" the whole stimulus "life." An explicit expression of this statement is the use of the introduced generalized moment of stimulus presentation (GMSP).

The third assumption is that the localization center processes the information about the locus of each stimulated point on the retina separately from every other point. Thus, the stimulus image on the retina is to be considered as a set of point-like images, and for each of them the theory for localization of a single stimulus is to be applied.

These assumptions might seem artificial and implausible. This holds true especially for the third assumption, because specific mechanisms exist for stripe detection which are not an assembly of point processors. The question is very complicated because, regardless of the existence of stripe detectors, the linear retinal trace in Experiment 3 leads to a perception of a curvilinear trace. More impressive examples of this kind can be found in the work of Kennard, Hartmann, Kraft, and Glaser (1971).

The present analysis does not concern the controversy about the possible origin of the ERS- "outflow" or "inflow" (Matin, 1972). It is important for our considerations that information about eye position is taken into account in the process of localization. In this work, we did not find any contradiction between this suggestion and the experimental data.

\section{REFERENCE NOTE}

1. Matin, E., Matin, L., Pola, J., \& Kowal, K. The intermittent light illusion and constancy of visual direction during voluntary saccades. Paper presented at the meetings of the Psychonomic Society, St. Louis, Missouri, 1969.

\section{REFERENCES}

Bischof, N., \& Kramer, E. Untersuchungen und Überlegungen zur Richtungswahrnehmung bei willkürliçhen sakkadischen Augenbewegungen. Psychologische Forschung, 1968, 32, 185-218.

HeNDry, D. P. Saccadic velocities determined by a new perceptual method. Vision Research, 1975, 15, 149-151.

Holly, F. Saccadic presentation of a moving target. Vision Research, 1975, 15, 331-335.

Holst, E. von. Relations between the central nervous system and the peripheral organs. British Journal of Animal Behavior. 1954, 2, 89-94.

Holst, E., von, \& Mittelstaedt, H. Das Reafferenzprinzip. Die Naturwissenschaften, 1950, 20, 464-476. 
Kennard, D. W., Hartmann, R. W., Kraft, D. P., \& Glaser, G. H. Brief conceptual (nonreal) events during eye movements. Biological Psychiatry, 1971, 3, 205-215.

Масн, E. Die Analyse der empfindungen. Jena, Germany: Fischer, 1885.

Matis, L. Eye movements and perceived visual direction. In Hurvich and Jameson (Eds.), Handbook of sensory physiology (Vol. VII/4) Visual psychophysics. Heidelberg, Germany: Springer, 1972.

Matin, L., \& Matin, E. Visual perception of direction and voluntary saccadic eye movements. Bibliotheca Ophthalmologica, 1972, 82, 358-368.

Matin, L., Matin, E., \& Pearce, D. C. Visual perception of direction when voluntary saccades occur. I. Relation of visual direction of a fixation target extinguished before a saccade to a flash presented during the saccade. Perception \& Psychophysics, 1969, 5, 65-80.

Matin, L., Matin, E., \& Pola, J. Visual perception of direction when voluntary saccades occur: II. Relation of visual direction of a fixation target extinguished before a saccade to a subsequent test flash presented before the saccade. Perception \& Psychophysics, 1970, 8, 9-14.

Matin, L., \& Pearce, D. G. Visual perception of direction for stimuli flashed during voluntary saccadic eye movements. Science, 1965, 148, 1485-1488.

Mitrani, L., Mateeff, S., \& Yakimoff, N. Smearing of the retinal image during voluntary saccadic eye movements. Vision Research, 1970, 10, 405-409.

Monahan, J. S. Extraretinal feedback and visual localization. Perception \& Psychophysics, 1972, 12, 394-353.

Sherrington, C. S. Observations on the sensual role of the proprioceptive nerve-supply of the extrinsic ocular muscles. Brain, 1918, 41, 323-343.

\section{NOTES}

1. The cathode-ray tube was of sufficiently short phosphor decay to produce flashes less than $1 \mathrm{msec}$. The duration of the flashes was determined by a photomultiplier.

2. The results of the present analysis (see Figure 5) will not change if both eyes are considered separately. Of course, a bilateral symmetry is to be assumed. In the case of asymmetry, double localizations (perceptions of two flashes) are expected to occur. There were no such reports in our experiments. Double localizations were observed by Bischof and Kramer (1968) and Kennard, Hartmann, Kraft, and Glaser (1971), who pointed out that they might be due to binocular vision.

3. "Saccadic suppression" provides a possible explanation of the perceptive shortening of the stimulus trace. Matin and Matin (1972) pointed out that such smears are, at least partially, suppressed. However, suppression seems not to act always. Hendry (1975) proposed a method for determination of saccadic velocity based on the perception of the stimulus trace. He did not report any suppression. On the other hand, simple observations of the "intermittent light illusion" show that the number of the perceived flashes of the "string" corresponds well to the saccade duration. Some of the flashes are of lower brightness, but, obviously, the shortening is due to distortions rather than to suppression. In any case, to avoid suppression, we used highly suprathreshold intensities of the stimuli.

(Received for publication January 11, 1978; revision accepted June 7,1978 .) 